\title{
Prediction of long-term variation in offspring metabolism due to BPA in eggs in rainbow trout using the DEB model
}

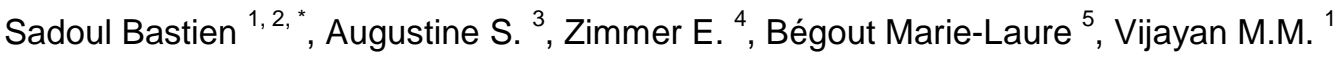 \\ ${ }^{1}$ Department of Biological Sciences, University of Calgary, Calgary, Alberta, Canada \\ ${ }^{2}$ MARBEC, Univ Montpellier, CNRS, Ifremer, IRD, Palavas-Les-Flots, France \\ ${ }^{3}$ Akvaplan-niva, Fram - High North Research Centre for Climate and the Environment, 9296 Tromsø, \\ Norway \\ ${ }^{4}$ Ibacon $\mathrm{GmbH}$, Arheilger Weg 17, 64380 Roßdorf, Germany \\ ${ }^{5}$ Ifremer, Laboratoire Ressources Halieutiques de La Rochelle, Place Gaby Coll, 17137 L'Houmeau, \\ France \\ * Corresponding author : Bastien Sadoul, email address : $\underline{\text { bastien.sadoul@ifremer.fr }}$
}

\begin{abstract}
:
Bisphenol A (BPA) in eggs prior to fertilization was found to induce long-term metabolic disturbances in juvenile rainbow trout (Oncorhynchus mykiss). Here we describe these imprinting effects in a Dynamic Energy Budget (DEB) framework, which allows interpretation of the data in an energy allocation context. First, DEB parameters for control rainbow trout were optimized using data extracted from 12 studies in the literature. Several modes of action on DEB parameters were then tested in order to correctly predict weight differences observed in rainbow trout in response to 5 different concentrations of BPA in eggs prior to fertilization. Reduced energy conductance $\dot{v}$ at day 0 , followed by an exponential recovery, was found to closely fit the experimental data. Effects on $\dot{v}$ lasted beyond the disappearance of the chemical from the body, suggesting an imprinting effect of BPA on energy mobilization from the reserve. Our model predicts that early changes in DEB parameters lead to permanent and irreversible impairment of the metabolic growth acceleration in rainbow trout.
\end{abstract}

\section{Highlights}

A control DEB model was obtained for rainbow trout using data extracted from 12 studies. Mode of action of BPA exposure prior fertilization was investigated at 5 different concentrations. BPA imprints DEB parameters long after being cleared from the animal. Impaired DEB parameters at early life stages lead to irreversible changes related to acceleration factor. 


\section{Introduction}

Rainbow trout (Oncorhynchus mykiss), a native species from North America, is highly adaptive to a wide range of environments and widely distributed across all continents. This species primarily resides in freshwater, while their anadromous form, called steelhead trout, are euryhaline and migrates from river to ocean (McCormick et al., 2013). Rainbow trout is at the top of the food chain and, therefore, of major ecological importance. They are also the main freshwater cultured fish species in Europe, with a total value of almost 3 billion USD in 2015 (FAO Fisheries and Aquaculture Department, 2017). Trout is therefore of key importance ecologically and economically, motivating numerous research programs in understanding the effect of environmental factors on fish performance, including growth, immune function and coping with stressor.

Bisphenol A (BPA) is a widely used industrial compound, especially in the manufacture of plastics and epoxy resins, and ubiquitously found in the aquatic environment. BPA is an endocrine-disrupting compound and affects various aspects of animal performance, including embryonic development (Birceanu et al., 2015b,a), growth (Ramakrishnan and Wayne, 2008) and sexual maturation (Sohoni et al., 2001; Mihaich et al., 2012; Ramakrishnan and Wayne, 2008). Recently, a study on egg exposure to BPA, mimicking maternal transfer of this contaminant, showed long-term effects on growth and gene expressions related to metabolism (Sadoul et al., 2017). This last study showed increased expression of genes related to protein degradation at 140 days post fertilization (dpf), whereas expression of genes related to lipid synthesis and most importantly cholesterol, were upregulated at $365 \mathrm{dpf}$. These results suggest that BPA has a long-term effect on energy re-allocation in rainbow trout.

Consequently, developing an integrative model to describe the rainbow trout life cycle in terms of energy allocation to different physiological processes may have value in predicting their ability to grow, reproduce and survive in a variable environment, including climate change, limited food availability, and impact of toxicants such as BPA (Nisbet et al., 2000). Additionally, from an aquaculture stand-point, effects of changes in rearing conditions or food compositions can be precisely estimated and characterized using such a model. The Dynamic Energy Budget (DEB) theory proposed by Kooijman (2010) is founded on the hypothesis that the life cycle of all living organisms share a common conceptual framework based on energy allocation starting from energy absorption to energy use for different physiological functions including growth, reproduction and maintenance. The parameters for DEB models were previously successfully estimated in aquatic species and used to describe sublethal effects due to chronic exposure to water contaminants (Ashauer and Jager, 2017). Recently, a DEB model was, for example, used to predict whether Pacific oysters, Crassostrea gigas, exposed to polystyrene microplastics increase not only the cost for growth, but also the cost for maintenance of structure, leading to a decrease in energy used for reproduction (Sussarellu et al., 2016). Similarly, a DEB model was used to reveal that uranium increases the cost for growth during the early life stages of zebrafish, Danio rerio (Augustine et al., 2012). However, so far, DEB model has not been utilized for rainbow trout 
in any scenario and more importantly never been used to predict long-term effects due to early developemental exposure to contaminants.

The aim of this study was to provide DEB parameters that best describes energy allocation over time of non-treated rainbow trout using literature-based data, and to describe the longterm effects of prenatal acute BPA exposure on DEB parameters in rainbow trout.

\section{Material and methods}

\subsection{Long-term BPA effects on growth in rainbow trout}

A recent publication by Sadoul et al. (2017) reported the long-term metabolic responses to BPA in eggs prior to fertilization. Reduced growth was observed in two experiments during the pre-juvenile stage followed by a sudden catch-up growth. The results suggested that these effects were related to increased proteolysis and decreased proteosynthesis in pre-juveniles, followed by increased production of lipids in post-juveniles. Growth data from this publication, along with data from Aluru et al. (2010), obtained under similar experimental conditions, were used in the present study. Protocols are briefly described below, but for further details please refer to the original publications (Sadoul et al., 2017; Aluru et al., 2010).

Rainbow trout eggs were exposed for 3 hours to ovarian fluid supplemented with either vehicle ( $<0.01 \%$ ethanol), $0.3,3,30,100$ or $300 \mathrm{mg} / \mathrm{L}$ of BPA. In the rest of the paper, treatments are respectively referred to as BPA0, BPA0.3, BPA3, BPA30, BPA100 and BPA300. Oocytes were then fertilized with untreated milt, rinsed with clean water, and incubated in separate trays. At $49 \mathrm{dpf}$, most of the trout hatched and by this time the BPA was also cleared from the animal (Aluru et al., 2010; Birceanu et al., 2015b). The larvae with yolk-sac were transfered to $200 \mathrm{~L}$ tanks receiving water at $8.5^{\circ} \mathrm{C}$ and fed exogenous food from $65 \mathrm{dpf}$ onwards. Automatic feeders distributed rations hourly and this was adjusted for each tank weekly based on the estimated tank biomass. The total amount of food was calculated using identical ration tables for all treatments and ranging from $4.5 \%$ of the body weight at the pre-juvenile stage to $0.5 \%$ when average fish weight was above $1 \mathrm{~kg}$. Because of the large number of treatments, only BPA0 and BPA100 had replicate tanks until $391 \mathrm{dpf}$, after wich they were consolidated into a single tank per treatment until the end of the experiment (1070 dpf). The same protocol was also repeated in a second experiment with triplicates of tanks, but only for $421 \mathrm{dpf}$ and with the controls (BPA0) and the two environmentally relevant treatments, BPA3 and BPA30 (Sadoul et al., 2017). The number of fish per tank was 277 to 299 larvae. Here on, the two experiments are referred to as experiment 1 and experiment 2, respectively. Body mass was periodically estimated by measuring the total mass divided by the number of fish in the tank. Growth data for BPA0, BPA3 and BPA30 treatments from both experiments were previously described in Sadoul et al. (2017), whereas growth data for BPA100 were reported in Aluru et al. (2010). The present study describes for the first time results for treatment BPA300.

\subsection{The DEB-tox modeling framework}


BPA effects were modeled in the context of the DEB theory as a change in a parameter of the DEB model. Therefore, it was necessary to develop first a DEB model for untreated rainbow trout (control DEB parameters) using data extracted from the literature. Control DEB parameters were applied on growth values for control treatments in the two experiments. For BPA treated fish, a toxicity module was coupled to the control DEB model. The overview of the model, input parameters, output and general method is presented in figure 1.

\subsection{Development of a DEB model for untreated rainbow trout}

\subsubsection{The DEB model for fish}

The growth and development of rainbow trout is modeled using the DEB model for fish (Kooijman et al., 2011; Sadoul and Vijayan, 2016). The model covers the full life-cycle of the animal from the start of development until death by aging. The DEB model scheme is present in figure 1. The equations of the DEB model with metabolic acceleration between birth and a transition phase, called "metamorphosis" in DEB theory (Kooijman and Lika, 2014), have been summarized previously for fish by Augustine et al. (2012).

DEB theory assumes that food is first stored in reserves before being used to fuel metabolic processes. At a given moment, only a fraction $\kappa$ of mobilized reserve compounds are used for growth and maintenance. The rest, $1-\kappa$ goes towards maturation or reproduction and maturity maintenance. Stage transitions (hatch, birth, ceasing of metabolic acceleration, puberty) occur at a fixed maturity level, that is after a certain amount of energy has been invested into maturation. Age and size, to a lesser extent, can differ at stage transitions (Augustine et al., 2011). Additionally, growth is considered isomorphic, and it is assumed that organisms invest in maturity and maturity maintenance until puberty after which the flux is routed towards reproduction (Kooijman, 2010). Many fish species show a metabolic acceleration between birth and metamorphosis (Kooijman, 2014). In a DEB context, this growth phase is characterized by surface area growing proportional to volume, the technical term is V1-morphic growth, where the 1 refers to the exponent (the iso-morph is a V2/3 morph). Associated weight corresponds to the sum of the weights of reserve ( $E$ in joules) and the formed structures (V in cubic centimeters). See supplementary materials for more details.

\subsubsection{Overview of the literature data}

In order to calibrate the untreated model, data have been extracted from peer-reviewed journal articles from the literature. Studies reporting multiple observations over time (socalled uni-variate data) and, most importantly, those reporting environmental conditions (temperature and feeding conditions), were selected. Observations for one time point (zerovariate data) were added to overcome any missing information. In total, data from 12 peerreviewed articles have been used. Univariate data: To establish a length / weight relationship, data from four studies were compiled (Chen et al., 2015; Davidson et al., 2014; Weatherley and Gill, 1981; Sumpter et al., 1991). Growth over time was included for the embryonic stage as well as the late juvenile and early adult stages (Davidson et al., 2014; Ninness et al., 2006; Yanik et al., 2002). Oxygen consumption as a function of length (Kieffer 
et al., 1998) as well as temperature (Wieser, 1985) was included. Hatching times at different temperatures were extracted from Velsen (1987). Zero-variate data: Observations on weight and age at birth and hatching at different temperatures were obtained from From and Rasmussen (1991), and age and weight at puberty were taken from Davidson et al. (2014). Detailed information on reproductive output is essential for determining the allocation fraction to soma $(\kappa)$. However, since rainbow trout has a long lifecycle (2-3 years), only very limited quantitative information on reproduction was available (Tyler et al., 1996). Some additional data on ultimate weight and length as well as lifespan were collected from the fishbase (Froese and Pauly, 2017), summarizing 3 studies (Love et al., 2005; Hugg, 1996; Ray and Robins, 1986) By default, a scaled functional response of $\mathrm{f}=1$ is assumed for experimental data, which relates to ad libitum feeding. Only if the experimental setup or the data suggest a lower food level, the scaled functional response is estimated. Note that the model has been calibrated using a large amount of data that define growth, as well as ontogenic development and metabolic activity. However, there is insufficient data available for defining the reproductive output (only a zero-variate data point on reproduction rate). Thus, predictions for reproductive output should be treated with caution when using the model for long-term simulation studies.

The above described data were used for the parametrization of the rainbow trout DEB model with metabolic acceleration between birth and puberty. Parameters are presented in Table 1 . Corresponding estimations are now available online on the Add-my-Pet (AmP) website : bio.vu.nl/thb/deb/deblab/add_my_pet/, Oncorhynchus mykiss, version 2017/10/30.

\subsection{Application to the BPA experiments data}

\subsubsection{Description of growth for controls}

In order to account for environmental differences between the two experiments described in 2.1, performed at different years, initial energy in the eggs $\left(E_{0}\right)$ and feeding levels $(f)$ were estimated based, respectively on weight at birth (first feeding) and growth of untreated fish. Using DEB parameters obtained in 2.3 and estimated $f$ and $E_{0}$, growth data for control tanks were simulated.

\subsubsection{The toxicity module for BPA treated fish}

Decreased assimilation $\left(\left\{\dot{p}_{A m}\right\}\right)$, increased specific cost for structure $\left(\left[E_{G}\right]\right)$ and increased somatic maintenance $\left(\left[\dot{p}_{M}\right]\right)$ are commonly considered as the main modes of action (MoA) for impaired growth of treated animals (see Ashauer and Jager, 2017, for a review). In the present study, the contribution of these processes as well as decreased energy conductance $(i$ ), directly related to energy mobilization from reserves, and altered allocation fraction to soma $(\kappa)$ were considered. Each physiological mode of action was tested following the same assumptions:

- For both experiments, treated fish were assumed to have experienced the same experimental conditions as control tanks. The two BPA experiments, 
performed with different broodstocks, may have different egg sizes, estimated from control individuals (see 2.4.1). Temperature was $8.5^{\circ} \mathrm{C}$. Food ration for BPA treated tanks were supposed as equal to the mean of food ration estimated for control tanks : $f_{\text {experiment1 }}$ and $f_{\text {experiment } 2 \text {. }}$

- The targeted parameter changes proportional to the dimensionless stress function s. So, if a $M o A$ is targeted then the change in $M o A^{*}=M o A(1+s)$, where $\mathrm{S}$ is the dimensionless stress function following for $\left[E_{G}\right]$ and $\left[\dot{p}_{M}\right]$ an exponential decrease :

$$
\frac{d}{d t} s=-s k_{r}
$$

With $\dot{k}_{r}$, the repair rate. For $\left\{\dot{p}_{A m}\right\}$ and $\dot{v}, \mathrm{~s}$ was inversed and transformed into percentages (by dividing by 100), in order to describe an exponential recovery curve.

- The initial intensity of the stress $\left(s_{i n i}\right)$ is specific to the level of exposure and non-negative, whereas $k_{r}$ is assumed to be identical among all treatments.

\subsubsection{Relative error calculation}

The performance of a model was evaluated by comparing predictions of the model and real data. The relative error $(\mathrm{RE})$ measures this difference:

$$
R E=\frac{1}{n} \sum_{i=1}^{n}\left|1-\frac{Y_{i}}{Y_{i}}\right|
$$

with $\mathrm{n}$ the number of real data, $Y_{i}$ the real value and $Y_{i}$ the predicted value from the model.

For a given mode of action, the mean of REs calculated for each treatment (MRE) was provided.

\subsection{Softwares}

The DEBtool software (DEB2017 release available at https://github.com/add-mypet/DEBtool_M/releases) for Matlab (Matlab R2014b) was used for estimation of control DEB parameters. DEBtool uses an improved version of the 'covariation method' (Lika et al., 2011) to optimize DEB parameters. The AmP estimation procedure used here is described in Marques et al. (2018a,b).

DEB parameters for $O$. mykiss were compared with that of other species in AmP (2018) using matlab package AmPtool (Marques et al., 2018a), which is freely available on the AmP GitHub repository.

DEB parameters were then transfered to R (v3.3.3) (R Core Team, 2017) using the 'R.Matlab' package (Bengtsson, 2016), and DEB estimations associated with the tox-module were computed using differential equations solved with the 'deSolve' package (Soetaert et al., 
2010). Optimization of toxic parameters, $s_{i n i}$ and $k_{r}$, were performed using default options of the 'optim' function in R to minimize MRE.

\section{Results}

\subsection{DEB control parameters or "Eco-physiology of the blank"}

Using data from the literature and default estimation procedure from the DEBtool, DEB parameters for rainbow trout are presented in Table 1.

Not enough energetic data were found on digestion and faecation efficiency, therefore, both $\kappa_{X}, \kappa_{P}$ were set to 0.8 and 0.1 , respectively, as described for the general animal in AmP.

Studies focusing on nutrition or feed efficiency in a DEB context, should further refine these parameters by adding relevant information to the parametrization. For the same reasons, maturity maintenance rate coefficient was set as 0.002 . No data was available regarding when rainbow trout's metabolism stops accelerating. However, the combination of both embryo and adult data, provides enough information to estimate metabolic acceleration in rainbow trout. Using the DEBtool parameter estimation routines, we were therefore able to estimate the maturity level at metamorphosis.

\subsection{Predicted body mass for control tanks in the two BPA experiments}

Based on weight at birth, initial reserve in the egg (at fertilization) was estimated for each experiment. Eggs from experiment 1 had lower estimated energy content than experiment 2, i.e. 604 and $644 \mathrm{~J}$ respectively. Additionally, estimations of $f$ values for each control tank in both experiments are given in Table 2. Using estimated $E_{0}, f$ values and DEB parameters for the rainbow trout, egg wet weight, and body wet weight, age and length at birth and at metamorphosis were predicted (Table 3). Additionally, growth for all control tanks was predicted (Figures 2a, 2b and 2c) and compared to real values (RE in Table 2).

\subsection{Mode of action of BPA exposure prior to fertilization}

First, an effect on assimilation rate was considered. This described the reduced growth in BPA treated fish, but was unable to describe the quick catch-up phenomenon observed in treated groups (Supplementary Figure 1). Similarly, modification of $\kappa$ led to a quick decrease in weight of treated groups compared to controls but was incapable of showing any catch up growth (Supplementary Figure 2). Therefore, these modes of action were excluded from possible affected parameters. On the other hand, increasing the cost for growth or increasing the somatic maintenance costs were both able to precisely predict a decrease in growth, as well as the catch-up phenomenon, similar to the observed values (Supplementary Figures 3 and 4). Optimized parameters and associated relative error (RE) are presented in Table 4. For both modes of action, development was accelerated and time at metamorphosis decreased, as detailed in Supplementary Tables 1 and 2. In addition, effects on $\left[E_{G}\right]$ or $\left[\dot{p}_{M}\right]$ at early stages modify $L_{b}$ and $L_{j}$ with consequences on the acceleration factor $s_{M}$. 
Finally, $\dot{v}$ was also found to properly describe the temporal pattern for weight in treated fish compared to controls (Figure 3). The increase of $i$ to reach control value delays the time of phase transitions, i.e. birth and metamorphosis (Table 5). A change in energy conductance $\dot{v}$ was found to produce the best predictions of weight differences for treated fish, with an MRE of 4.63. This mode of action describes a linear dose-response effect of BPA as shown in figure 4 .

\section{Discussion}

The DEB theory provides a metabolic framework to describe and predict growth, development and reproduction over the full life cycle of rainbow trout in a given environment characterized by the amount of food available and the temperature of the water. The model, proposed here, accurately fits a wide range of physiological processes observed in the literature. This study further extends the number of fish species described in a DEB context (Sadoul and Vijayan, 2016). This model also highlights particularities in rainbow trout's metabolic processes compared to other fish species worth discussing.

\subsection{Control DEB parameters}

Parametrization of the DEB parameters using data from 12 studies, and information from fishbase, showed a good agreement between predictions and data from the literature (see supplementary Table 3 and supplementary Figure 5 for the model fits). Herein, we briefly discuss the fit of the model to the data highlighting strengths, weaknesses and some new questions. Overall, we found little quantitative information on reproduction in terms of numbers of eggs produced at varying food and temperatures. The maximum reproduction rate of 174 eggs. $d^{-1}$ at $12^{\circ} \mathrm{C}$ from Tyler et al. (1996) was used. Davidson et al. (2014) reported growth up to $5 \mathrm{~kg}$, and onset of sexual maturity under controlled laboratory conditions. The DEB model predicts well the weight and time at sexual maturity as well as the maximum reproduction (see supplementary Table 3). However, the DEB model did not explain why sexually mature fish in Davidson et al. (2014) abruptly stopped allocating energy to growth and why meat quality suddenly changed. Future studies might include more details on the reproductive physiology of rainbow trout and determine if observed values in Davidson et al. (2014) relates to rainbow trout in general or to rainbow trout reared in specific captivity conditions. Getting further insights on conditions affecting maturity is central for salmonid aquaculture industry as maturing fish have lower meat quality (Aksnes et al., 1986).

In addition, the model predicts extremely well the timing and weights at hatch and birth at different temperatures, as well as yolk and yolk-free dry mass during embryo development, as reported in Ninness et al. (2006). Using control parameters in Table 1, we were able to obtain a mechanistic interpretation of growth in the control groups in the BPA experiments. The fact that the model fits with the empirical data obtained for embryo development so well, allows us to infer differences in egg sizes between experiments 1 and 2 based on the differences in weight observed on day 64, which corresponds with the time of first-feeding (Table 3). We found that fish in experiment 1 were from smaller eggs compared to experiment 2 , but were better fed, with a food level of 0.7 compared to 0.6 in experiment 2. Our model also predicted 
that, overall, younger fish had a lower food level than older fish, as the estimated food level $f$ was almost 0.8 after $350 \mathrm{dpf}$ (Table 2).

It is always possible to obtain a good fit of the data, but DEB parameters might be unrealistic, implying unreasonable biological properties. Therefore, we evaluated our DEB parameter set not only in terms of goodness of fit, but also in terms on biological realism. This second form of evaluation is referred to as 'estimation-in-context' (Marques et al., 2018b). The parameters of $O$. mykiss (Table 1) were discussed with respect to DEB parameters of other Salmoniformes and Actinopterygii available in AmP (AmP, 2018, 12/15/2017).

\subsection{Rainbow trout metabolism in context}

O. mykiss is part of the Salmoniformes, which are part of the ray-finned fish group (Actinopterygii). We restricted the comparison to ray-finned fish, anatomically similar to $O$. mykiss.

We looked at how DEB parameters presented in Table 1 were distributed across Actinopterygii $(n=200)$ and Salmoniformes $(n=7)$ following the approach described in Kooijman and Lika (2014). Parameters for all species are plotted as function of their ultimate size $L_{i}$ (Lika et al., 2018). The parameter estimation method used in the present study operates with pseudo-data, providing a priori information about parameter values. These pseudo-data refer to parameters of a generalized animal and are expected to covary in specific ways with size (see Kooijman and Lika, 2014). Expectations based on the values for the generalized animal and the covariation rules are presented as a grey line in Figure 5. In addition, to compare DEB parameters between species we also compared some relevant quantities, which are functions of DEB model parameters (Figure 5): the maximum reserve density $\left[E_{m}\right]=\dot{p}_{A m} / \dot{v}$ in $\mathrm{J} / \mathrm{cm}^{3}$, the maximum respiration rate in $\mathrm{mol}_{2} / \mathrm{d}$, as well as the mean

life span $a_{m} \approx 1.62\left(\frac{\kappa\left\{\dot{p}_{A m}\right\}}{h_{a} \dot{v}\left[\dot{p}_{M}\right]}\right)^{1 / 3},($ Kooijman, 2010).

Overall, O. mykiss has a remarkably high $\left\{\dot{p}_{A m}\right\}$ and $\left[\dot{p}_{M}\right]$ compared to values estimated for other fish species, while the value of $i$ was more typical (Figure 5 top row). Maturity levels were not outliers with respect to that of other fish species (Figure 5 middle row). The maximum reserve density was high with respect to other species of this size, as well as with respect to other Salmoniformes. Mean life-span was inversely proportional to somatic maintenance in many taxa (Lika et al., 2018), and the relationship was relatively clear among fishes (Figure 5 bottom row). The high $\left\{\dot{p}_{A m}\right\}$ was responsible for the high reserve density, translating a probable good storage capacity, while the high $\left[\dot{p}_{M}\right]$ was responsible for the low mean life span. That being said, other species of similar $\left[\dot{p}_{M}\right]$ have a much longer life-span, and those species were able to do that by having low reserve density. The expected maximum respiration of $O$. mykiss with respect to its size was within the range of other fish species. In summary DEB parameters for rainbow trout are not extreme outliers and are biologically realistic. $O$. mykiss mainly distinguishes itself by its large reserve capacity. 


\subsection{Mode of action of imprinting effects of BPA}

The control DEB model, parametrized using data from the literature, accurately fits growth data of control tanks presented in Sadoul et al. (2017), when $f$ is the only free parameter. Estimation of $f$ for control tanks, suggests that fish from experiments 1 and 2 were assimilating during the same period approximately 70 and $60 \%$ of the maximum assimilation rate, respectively.

The long-term effects of BPA on weight reveal a decreasing weight difference until $260 \mathrm{dpf}$ for all BPA treatments above $3 \mathrm{mg} / \mathrm{L}$, and a sudden catch-up phenomenon after $260 \mathrm{dpf}$. This phenomenon was previously described for BPA30 treatment (Sadoul et al., 2017), but is reinforced here by additional BPA concentrations. The decrease in body mass seems to be concentration-dependent with BPA300 showing the strongest difference to that of the control. The three MoA able to describe impairment of growth in early stages and catch-up phenomenon in post juveniles $\left(\left[\dot{p}_{M}\right], E_{G}\right.$ and $\left.\dot{v}\right)$, were also able to capture the concentrationdependent effects of BPA. The choice of the correct MoA is often difficult, especially when the study is lacking additional data to weight or reproduction. Untangling an effect on maintenance cost or assimilation is, for example, particularly difficult when additional data on oxygen consumption are not available. Consequently, modeling work can fit equally well different MoA for a same contaminant, as observed for the effect of uranium on aquatic species (Augustine et al., 2012; Massarin et al., 2011; Goussen et al., 2015). In our study, the impact of BPA on $\dot{v}$ is the most suitable mode of action as it yields predictions particularly close to real values. Parameter $\dot{v}$ controls the speed of reserve mobilization (Kooijman, 2010). Decreased $i$, as observed for BPA treated groups, leads to a reduction of the amount of reserves mobilized. This is in agreement with results from Aluru et al. (2010), describing a delay in the use of the yolk sac in BPA treated eggs. In addition, decreased $\dot{v}$ at early stages leads to less energy allocated to maturity and growth. This has for consequences to slow down development and to reduce the time taken for stage transitions (birth and metamorphosis). Although developmental effect was not significant at lower BPA concentrations (Birceanu et al., 2015b; Sadoul et al., 2017), Aluru et al. (2010) reported delays in hatching and time at first feeding for fish from eggs treated with high concentrations of BPA. This is in accordance with various delays in maturity or development reported in terrestrial model species (Tan et al., 2003; Atlı and Ünlü, 2012; Hijazi et al., 2015). However, previous results also showed opposite effects of BPA exposure during ontogeny in medaka, Oryzias latipes, a species with a short life cycle (Ramakrishnan and Wayne, 2008).

In the present study, to fit effects of exposure to BPA prior to fertilization, impairment of $i$ is required to have a long mean lifetime (1/k, 154.4 days). Assuming that BPA concentration in treated fish is absent by $40 \mathrm{dpf}$, the repair rate of $v$ could be attributed to dilution of damaged compounds by structures or reserves. But in both cases, even if we assume that elimination is null, dilution is too fast to fit reconstructed $\dot{v}$ over time. If the change in $\dot{v}$ was directly linked to the internal concentration of BPA in the organism (which is often a realistic assumption in DEB toxicological applications (Jager and Zimmer, 2012)), the effect on $\dot{v}$ would be reversed much faster. Therefore, the relatively slow depuration of the effect, despite dilution by 
growth, suggests existence of a system of proliferation or propagation of toxicological effects across new compounds in structure and reserve. This adds to the understanding of potential mechanisms of action of BPA exposure during early life stages. Epigenetic modifications are good candidates for the mechanisms of action of BPA, because they are described as stable over mitosis, allowing toxicological effects to last despite disappearance of toxic compounds (Skinner, 2011, for a review on the mechanisms). Indeed, epigenetic alterations are inherited by daughter cells during mitosis, implying that modification of the epigenome during development can be transmitted to that cells lineage, with potential long-term consequences on associated tissue.

\subsection{Suggested effects on the acceleration factor and implications}

To our knowledge this is the first time that the effect of a compound could be explained by assuming an effect on the acceleration factor $s_{M}$. Our findings can support the theory underlying the metabolic acceleration, and help uncover its metabolic function. This effect is moderate (2.65 for BPA300 versus 2.61 for the control) in the present study, but has several important implications worth discussing.

First, since $s_{M}$ is the ratio of length at metamorphosis and length at birth, the value of $s_{M}$ becomes fully fixed at metamorphosis. This implies that a change of $s_{M}$, similarly to what is observed in treated groups in our study, is irreversible irrespective of the environment. Even if the compound is eliminated from the body, or even if the animal is exposed after metamorphosis to a compound with opposed effects, the change in acceleration factor will remain. In BPA300 fish, $s_{M}$ was increased compared to controls, with the consequences that treated individuals have increased assimilation of energy and increased reserve mobilization in an identical environment. The ultimate length can therefore be expected to be longer for treated individuals. As a consequence, in such a scenario, ultimate size of treated individuals will exceed their genetically determined target size. The underlying biological mechanisms still need to be understood, but increased nutrient absorption or digestion efficiency may play a role, and should be considered as potential candidates for explaining the obesogenic activity of BPA (vom Saal et al., 2012).

\subsection{Conclusions}

We present here a detailed model of energy allocation for rainbow trout based on the DEB theory. Using this model, we demonstrate that BPA imprints DEB parameters in early stages with a repair rate slower than dilution by growth, suggesting a propagating effect of the damage. The change in $\dot{v}$ during early stages leads to a shift in the timing of birth and metamorphosis, impacting $s_{M}$ and thereby leading to persistent and irreversible metabolic changes. Being able to model imprinting effects of contaminants using the DEB metabolic framework can help elucidate the effect of contaminants at the population level and predict effects in a new environment with variable food and temperature regimes.

\section{Acknowledgements}


This study was supported by the Natural Sciences and Engineering Research Council of Canada (NSERC) Discovery and Strategic Project Grants to Dr. Vijayan. Dr. Augustine was supported by the Norwegian Science Council (NFR 255295, "Integrating eco-physiological $\&$-toxicological data into marine ecosystem-based management"). Dr. Sadoul received a visiting researcher grant from the Norwegian Science Council (NFR 268241). We thank Drs. Aluru and Birceanu for providing the data from their BPA experiments for this study, and the personnel at the Alma Research Station, Ontario, Canada, for breeding and maintaining the trout. 


\section{References}

(2018). Add-my-pet. http://www.bio.vu.nl/thb/deb/deblab/add \_my\_pet/.

Aksnes, A., Gjerde, B., and Roald, S. O. (1986). Biological, chemical and organoleptic changes during maturation of farmed Atlantic salmon, Salmo salar. Aquaculture, 53(1):7-20.

Aluru, N., Leatherland, J. F., and Vijayan, M. M. (2010). Bisphenol A in Oocytes Leads to Growth Suppression and Altered Stress Performance in Juvenile Rainbow Trout. PLoS ONE, 5(5):e10741.

Ashauer, R. and Jager, T. (2017). Physiological modes of action across species and toxicants: The key to predictive ecotoxicology. Environmental Science: Processes \& Impacts.

Atl1, E. and Ünlü, H. (2012). Developmental and reproductive effects of bisphenol A (Bpa) in Drosophila Melanogaster. Hacettepe J Biol Chem, 40:61-68.

Augustine, S., Gagnaire, B., Adam-Guillermin, C., and Kooijman, S. A. L. M. (2012). Effects of uranium on the metabolism of zebrafish, Danio rerio. Aquatic Toxicology, 118-119:9-26.

Augustine, S., Gagnaire, B., Floriani, M., Adam-Guillermin, C., and Kooijman, S. A. L. M. (2011). Developmental energetics of zebrafish, Danio rerio. Comparative Biochemistry and Physiology Part A: Molecular \& Integrative Physiology, 159(3):275-283.

Bengtsson, H. (2016). R.matlab: Read and Write MAT Files and Call MATLAB from Within R. R package version 3.6.1.

Birceanu, O., Mai, T., and Vijayan, M. M. (2015a). Maternal transfer of bisphenol A impacts the ontogeny of cortisol stress response in rainbow trout. Aquatic Toxicology, 168:11-18.

Birceanu, O., Servos, M. R., and Vijayan, M. M. (2015b). Bisphenol A accumulation in eggs disrupts the endocrine regulation of growth in rainbow trout larvae. Aquatic Toxicology, 161:51-60.

Chen, Z., Snow, M., Lawrence, C. S., Church, A. R., Narum, S. R., Devlin, R. H., and Farrell, A. P. (2015). Selection for upper thermal tolerance in rainbow trout (Oncorhynchus mykiss Walbaum). Journal of Experimental Biology, 218(5):803-812.

Davidson, J. W., Kenney, P. B., Manor, M., Good, C. M., Weber, G. M., Aussanasuwannakul, A., Turk, P. J., Welsh, C., and Summerfelt, S. T. (2014). Growth performance, fillet quality, and reproductive maturity of Rainbow Trout (Oncorhynchus mykiss) cultured to 5 kilograms within freshwater recirculating systems. Journal of Aquaculture Research and Development, 5(4).

FAO Fisheries and Aquaculture Department (2017). FAO. 2017. Fishery and Aquaculture Statistics. Global aquaculture production 1950-2015 (FishstatJ).

Froese, R. and Pauly, D. (2017). FishBase 2017, version (june, 2017). World Wide Web electronic publication. Home page at: http://www. fishbase. org. 
From, J. and Rasmussen, G. (1991). Growth of rainbow trout, Oncorhynchus mykiss (Walbaum, 1792) related to egg size and temperature. Dana, 9:31-38.

Goussen, B., Pery, A. R., Bonzom, J., and Beaudouin, R. (2015). Transgenerational adaptation to pollution changes energy allocation in populations of nematodes. Environmental Science and Technology.

Hijazi, A., Guan, H., Cernea, M., and Yang, K. (2015). Prenatal exposure to bisphenol A disrupts mouse fetal lung development. FASEB journal: official publication of the Federation of American Societies for Experimental Biology, 29(12):4968-4977.

Hugg, D. O. (1996). MAPFISH georeferenced mapping database. Freshwater and estuarine fishes of North America. Life Science Software. Dennis O. and Steven Hugg, 1278.

Jager, T. and Zimmer, E. I. (2012). Simplified Dynamic Energy Budget model for analysing ecotoxicity data. Ecological Modelling, 225:74-81.

Kieffer, J. D., Alsop, D., and Wood, C. M. (1998). A respirometric analysis of fuel use during aerobic swimming at different temperatures in rainbow trout (Oncorhynchus mykiss). Journal of Experimental Biology, 201(22):3123-3133.

Kooijman, S. A. L. M. (2010). Dynamic Energy Budget Theory for Metabolic Organisation. Cambridge University Press, Cambridge, United Kingdom.

Kooijman, S. A. L. M. (2014). Metabolic acceleration in animal ontogeny: An evolutionary perspective. Journal of Sea Research, 94(Supplement C):128-137.

Kooijman, S. A. L. M. and Lika, K. (2014). Comparative energetics of the 5 fish classes on the basis of dynamic energy budgets. Journal of Sea Research, 94:19-28.

Kooijman, S. A. L. M., Pecquerie, L., Augustine, S., and Jusup, M. (2011). Scenarios for acceleration in fish development and the role of metamorphosis. Journal of Sea Research, 66(4):419-423.

Lika, K., Augustine, S., and Kooijman, S. A. L. M. (2018). Body size as emergent property. J. Sea Res., this issue.

Lika, K., Kearney, M. R., and Kooijman, S. A. L. M. (2011). The "covariation method" for estimating the parameters of the standard Dynamic Energy Budget model II: Properties and preliminary patterns. Journal of Sea Research, 66(4):278-288.

Love, M. S., Mecklenburg, C. W., Mecklenburg, T. A., and Thorsteinson, L. K. (2005). Resource inventory of marine and estuarine fishes of the West Coast and Alaska: A checklist of North Pacific and Arctic Ocean species from Baja California to the Alaska - Yukon border. Usgs unnumbered series, U.S Geological Survey. 
Marques, G., Augustine, S., Lika, K., Pecquerie, L., Domingos, T., and Kooijman, S. (2018a). The amp project: Comparing species on the basis of dynamic energy budget parameters. PLOS Computational Biology. submitted.

Marques, G., Lika, K., Augustine, S., Pecquerie, L., and Kooijman, S. (2018b). Fitting multiple models to multiple data. Journal of Sea Research. submitted to this special issue.

Massarin, S., Beaudouin, R., Zeman, F., Floriani, M., Gilbin, R., Alonzo, F., and Pery, A. R. R. (2011). Biology-based modeling to analyze uranium toxicity data on daphnia magna in a multigeneration study. Environmental Science \& Technology, 45(9):4151-4158.

McCormick, S. D., Farrell, A. P., and Brauner, C. J. (2013). Fish Physiology: Euryhaline Fishes. Academic Press.

Mihaich, E., Rhodes, J., Wolf, J., van der Hoeven, N., Dietrich, D., Hall, A. T., Caspers, N., Ortego, L., Staples, C., Dimond, S., and Hentges, S. (2012). Adult fathead minnow, Pimephales promelas, partial life-cycle reproductive and gonadal histopathology study with bisphenol A. Environmental Toxicology and Chemistry, 31(11):2525-2535.

Ninness, M. M., Stevens, E. D., and Wright, P. A. (2006). Removal of the chorion before hatching results in increased movement and accelerated growth in rainbow trout (Oncorhynchus mykiss) embryos. Journal of Experimental Biology, 209(10):1874-1882.

Nisbet, R. M., Muller, E. B., Lika, K., and Kooijman, S. a. L. M. (2000). From molecules to ecosystems through dynamic energy budget models. Journal of Animal Ecology, 69(6):913926.

R Core Team (2017). R: A Language and Environment for Statistical Computing. $\mathrm{R}$ Foundation for Statistical Computing, Vienna, Austria.

Ramakrishnan, S. and Wayne, N. L. (2008). Impact of bisphenol-A on early embryonic development and reproductive maturation. Reproductive Toxicology, 25(2):177-183.

Ray, C. and Robins, C. R. (1986). A Field Guide to Atlantic Coast Fishes: North America. Houghton Mifflin Harcourt.

Sadoul, B., Birceanu, O., Aluru, N., Thomas, J. K., and Vijayan, M. M. (2017). Bisphenol A in eggs causes development-specific liver molecular reprogramming in two generations of rainbow trout. Scientific Reports, 7(1):14131.

Sadoul, B. and Vijayan, M. M. (2016). 5 - Stress and Growth. In Schreck, A. P. F. and Colin J. Brauner Carl B., L. T., editors, Fish Physiology, volume 35 of Biology of Stress in FishFish Physiology, pages 167-205. Academic Press.

Skinner, M. K. (2011). Role of epigenetics in developmental biology and transgenerational inheritance. Birth Defects Research Part C: Embryo Today: Reviews, 93(1):51-55. 
Soetaert, K., Petzoldt, T., and Setzer, R. W. (2010). Solving Differential Equations in R: Package deSolve. Journal of Statistical Software, 33(9):1-25.

Sohoni, P., Tyler, C. R., Hurd, K., Caunter, J., Hetheridge, M., Williams, T., Woods, C., Evans, M., Toy, R., Gargas, M., and Sumpter, J. P. (2001). Reproductive Effects of LongTerm Exposure to Bisphenol A in the Fathead Minnow (Pimephales promelas). Environmental Science \& Technology, 35(14):2917-2925.

Sumpter, J. P., Le Bail, P. Y., Pickering, A. D., Pottinger, T. G., and Carragher, J. F. (1991). The effect of starvation on growth and plasma growth hormone concentrations of rainbow trout, Oncorhynchus mykiss. General and Comparative Endocrinology, 83(1):94-102.

Sussarellu, R., Suquet, M., Thomas, Y., Lambert, C., Fabioux, C., Pernet, M. E. J., Goïc, N. L., Quillien, V., Mingant, C., Epelboin, Y., Corporeau, C., Guyomarch, J., Robbens, J., PaulPont, I., Soudant, P., and Huvet, A. (2016). Oyster reproduction is affected by exposure to polystyrene microplastics. Proceedings of the National Academy of Sciences, 113(9):2430 2435.

Tan, B. L. L., Kassim, N. M., and Mohd, M. A. (2003). Assessment of pubertal development in juvenile male rats after sub-acute exposure to bisphenol A and nonylphenol. Toxicology Letters, 143(3):261-270.

Tyler, C. R., Pottinger, T. G., Santos, E., Sumpter, J. P., Price, S. A., Brooks, S., and Nagler, J. J. (1996). Mechanisms controlling egg size and number in the rainbow trout, Oncorhynchus mykiss. Biology of Reproduction, 54(1):8-15.

Velsen, F. P. J. (1987). Temperature and Incubation in Pacific Salmon and Rainbow Trout: Compilation of Data on Median Hatching Time, Mortality and Embryonic Staging. Department of Fisheries and Oceans, Fisheries Research Branch, Pacific Biological Station. vom Saal, F. S., Nagel, S. C., Coe, B. L., Angle, B. M., and Taylor, J. A. (2012). The estrogenic endocrine disrupting chemical bisphenol A (BPA) and obesity. Molecular and Cellular Endocrinology, 354(1):74-84.

Weatherley, A. H. and Gill, H. S. (1981). Recovery growth following periods of restricted rations and starvation in rainbow trout Salmo gairdneri Richardson. Journal of Fish Biology, 18(2):195-208.

Wieser, W. (1985). Developmental and metabolic constraints of the scope for activity in young rainbow trout (Salmo Gairdneri). Journal of Experimental Biology, 118(1):133-142.

Yanik, T., Hisar, S. A., and Bölükbasi, C. (2002). Early Development And Growth Of Arctic Charr (Salvelinus Alpinus) And Rainbow Trout (Oncorhynchus Mykiss) At A Low Water Temperature. Israeli Journal of Aquaculture. 
Figure 1 Overview of the DEB-tox modeling framework used to interpret long-term sublethal effects of BPA accumulation in the egg of rainbow trout. Effects are simulated as a change in a parameter value of the DEB model, see model scheme in the central panel. In this scheme the boxes represent the state variables of the model and the arrows represent energy/mass fluxes of the different metabolic processes. Each process is quantified by a control DEB parameter, estimated from the literature data. BPA disrupts one of the parameter of the DEB model. The magnitude with which the parameter is modified as well as the rate of repair are determined by values of the two last input parameters (initial stress and repair rate). The experimental food level and initial egg size are obtained from the control data of the BPA experiments. The DEB model was run using the input parameters, and weight as function of age was calculated. The final output was a comparison between expected weight of treated fish compared to control fish. We estimated the initial stress associated with the different BPA treatments, as well as the BPA repair rate by minimizing the distance between the output and actual toxicity data.

Figure 2 Body mass predictions (red line) and empirical data (black dots) for untreated rainbow trout. DEB parameters in table 1 were used for the predictions. In experiment 1 , three tanks of untreated fish were reared separately until $391 \mathrm{dpf}$ (a) and then pooled together in a tank and reared until $1070 \mathrm{dpf}$ (b). In experiment 2, three tanks of untreated fish were reared up to $421 \mathrm{dpf}(\mathrm{c})$. Data and predictions are at $8.5^{\circ} \mathrm{C}$. Mean $f$ values calculated from table 2 were used for predictions : 0.70 (a), 0.78 (b) and 0.59 (c).

Figure 3 Simulation of BPA effects on growth using a DEB model with $\dot{v}$ increasing following an exponential recovery curve. Empirical data for differences in body mass between treated fish and control fish are represented by points for both experiments (a and c). Simulations (lines) are performed using $v$ increasing over time as illustrated in $\mathrm{b}$ and $\mathrm{d}$ and parameters in tables 1 and 2.

Figure 4 Initial intensity of the stress $\left(s_{i n i}\right)$ for each BPA treatment. A linear dose-response curve was fitted (black).

Figure 5 Metabolism of $O$. mykiss in the wider context of that of Salmoniformes and Actinopterygii. Symbols: small black dots: Actinopterygii; blue circles: Salmoniformes; blue square: O. mykiss. Figures generated using data from $\operatorname{AmP}(2018,12 / 15 / 2017)$, and AmPtool software (Marques et al., 2018a). Top two rows: DEB parameters as function of ultimate structural length. See table 1 for the interpretation and the units of each DEB parameter. Postmetamorphic parameter values for energy conductance $i$ and maximum surface area specific assimilation rate $\left\{\dot{p}_{A m}\right\}$ are plotted (see text for explanation). The empty square symbol represent the embryo value for $\dot{v}$ and $\left\{\dot{p}_{A m}\right\}$. Grey line: inter-specific scaling relationships assuming parameters of the generalized animal (Kooijman and Lika, 2014), see text, section 4.2 for explanation. Third row: maximum reserve density as function of ultimate length, maximum respiration as function of ultimate dry weight, and mean life span as function of maximum volume linked somatic maintenance. All of the quantities in this figure are calculated assuming ad libitum food and reference temperature $\left(20^{\circ} \mathrm{C}\right)$. 
Table 1 Primary parameter values estimated in this study at reference temperature $\left(20^{\circ} \mathrm{C}\right)$ and uploaded online (AmP version 20171030).

\begin{tabular}{|c|c|c|c|}
\hline Symbol & Value & Unit & Description \\
\hline$\left\{\dot{p}_{A m}\right\}$ & 2512 & $\mathrm{~J} / \mathrm{d} / \mathrm{cm}^{2}$ & $\begin{array}{l}\text { maximum surface area } \\
\text { specific assimilation } \\
\text { rate }\end{array}$ \\
\hline$\left\{F_{m}\right\}$ & 6.5 & $\mathrm{l} / \mathrm{d} / \mathrm{cm}^{2}$ & $\begin{array}{l}\text { maximum specific } \\
\text { searching rate }\end{array}$ \\
\hline$K_{x}$ & 0.8 & - & $\begin{array}{l}\text { digestion efficiency of } \\
\text { food to reserve }\end{array}$ \\
\hline$K_{P}$ & 0.1 & - & $\begin{array}{l}\text { faecation efficiency of } \\
\text { food to faeces }\end{array}$ \\
\hline$\dot{v}$ & 0.03 & $\mathrm{~cm} / \mathrm{d}$ & energy conductance \\
\hline$\kappa$ & 0.62 & - & $\begin{array}{l}\text { allocation fraction to } \\
\text { soma }\end{array}$ \\
\hline$\kappa_{R}$ & 0.95 & - & reproduction efficiency \\
\hline$\left[\dot{p}_{M}\right]$ & 344 & $\mathrm{~J} / \mathrm{d} /$ & $\begin{array}{l}\text { volume specific } \\
\text { somatic maintenance }\end{array}$ \\
\hline$\dot{k_{J}}$ & 0.002 & & $\begin{array}{l}\text { maturity maint rate } \\
\text { coefficient }\end{array}$ \\
\hline$\left[E_{G}\right]$ & 5268 & $\mathrm{~J} / \mathrm{cm}^{3}$ & $\begin{array}{l}\text { specific cost for } \\
\text { structure }\end{array}$ \\
\hline$E_{H}^{b}$ & 43 & $\mathrm{~J}$ & maturity at birth \\
\hline$E_{H}^{j}$ & 854 & $\mathrm{~J}$ & $\begin{array}{l}\text { maturity at } \\
\text { metamorphosis }\end{array}$ \\
\hline$E_{H}^{p}$ & $38810^{4}$ & $\mathrm{~J}$ & maturity at puberty \\
\hline$h_{a}$ & 3.004 & $1 / d^{2}$ & $\begin{array}{l}\text { Weibull aging } \\
\text { acceleration }\end{array}$ \\
\hline$s_{G}$ & 10 & - & $\begin{array}{l}\text { Gompertz stress } \\
\text { coefficient }\end{array}$ \\
\hline
\end{tabular}

Parameters $\left\{\dot{F}_{m}\right\}, \kappa_{X}, \kappa_{P}, \kappa_{R}, \dot{k}_{J}, \ddot{h_{a}}$ and $s_{G}$ were fixed. 
Table 2 Scaled functional response (f) estimated for control tanks in the two BPA experiments. Relative error (RE) is provided for each tank. In experiment 1 , three tanks of untreated fish were reared separately until $391 \mathrm{dpf}$ and then pooled together in a tank and reared until $1070 \mathrm{dpf}$ (b). In experiment 2, three tanks of untreated fish were reared up to 421 dpf.

\begin{tabular}{|l|l|l|l|l|}
\hline Experiment & $f$ & RE & Tank & \\
\hline 1 & 0.72 & 0.09 & A & \\
\hline & 0.66 & 0.07 & B & \\
\hline & 0.71 & 0.07 & C & \\
\hline & 0.78 & 0.05 & pooled & \\
\hline 2 & 0.58 & 0.12 & A & \\
\hline & 0.59 & 0.10 & B & \\
\hline & 0.58 & 0.12 & C & \\
\hline & & & & \\
\hline
\end{tabular}


Table 3 Egg weight, age at birth, age at metamorphosis, size at birth, size at metamorphosis, acceleration factor as predicted using control DEB parameters at $8.5^{\circ} \mathrm{C}$

\begin{tabular}{|c|c|c|c|c|c|}
\hline & Experiment 1 & Experiment 2 & ad libitum & description & unit \\
\hline$E_{0}$ & 604 & 644 & 887 & energy in egg & J \\
\hline$f$ & 0.7 & 0.6 & 1 & $\begin{array}{l}\text { scaled } \\
\text { functional } \\
\text { response }\end{array}$ & - \\
\hline$W_{w}{ }^{0}$ & 0.1313 & 0.1398 & 0.1928 & $\begin{array}{l}\text { egg wet } \\
\text { weight }\end{array}$ & $\mathrm{g}$ \\
\hline$W_{w}^{b}$ & 0.1162 & 0.1248 & 0.1778 & $\begin{array}{l}\text { wet weight at } \\
\text { birth }\end{array}$ & $\mathrm{g}$ \\
\hline$W_{w}^{j}$ & 2.2402 & 1.9244 & 3.1855 & $\begin{array}{l}\text { wet weight at } \\
\text { metamorphosi } \\
\mathrm{s}\end{array}$ & $\mathrm{g}$ \\
\hline$a_{b}$ & 63.7487 & 63.5638 & 62.8118 & age at birth & d \\
\hline$a_{j}$ & 135.4721 & 138.1077 & 130.8014 & $\begin{array}{l}\text { age at } \\
\text { metamorphosi } \\
\mathrm{s}\end{array}$ & $d$ \\
\hline$L_{b}$ & 0.2146 & 0.2147 & & $\begin{array}{l}\text { structural } \\
\text { length at } \\
\text { birth, }\end{array}$ & $\mathrm{cm}$ \\
\hline$L_{j}$ & 0.5598 & 0.5578 & 0.5634 & $\begin{array}{l}\text { structural } \\
\text { length at } \\
\text { metamorphosi } \\
\mathrm{s}\end{array}$ & $\mathrm{cm}$ \\
\hline$s_{\mathcal{M}}$ & 2.6 & 2.6 & 2.6167 & $\begin{array}{l}\text { acceleration } \\
\text { factor }\end{array}$ & - \\
\hline & & 2 & & & \\
\hline
\end{tabular}


Table 4 Mode of actions and optimized parameters for BPA treated fish. The mean relative error (MRE) was calculated for each mode of action, by comparing predicted differences of weight to control with real differences (Figure 3).

\begin{tabular}{|l|l|l|l|l|l|}
\hline $\begin{array}{l}\text { Mode of } \\
\text { action }\end{array}$ & $\dot{k}_{r}\left(\mathrm{~d}^{-1}\right)$ & Treatment & $s_{\text {ini }}(-)$ & MRE & \\
\hline$\left[\dot{p}_{M}\right]$ & 0.0122 & BPA0.3 & 8.0 & 6.39 & \\
\hline & & BPA3 & 6.2 & & \\
\hline & & BPA30 & 9.1 & & \\
\hline & & BPA100 & 14.6 & & \\
\hline & & BPA300 & 25.8 & & \\
\hline$\left[E_{G}\right]$ & 0.0064 & BPA0.3 & 0.4 & 6.26 & \\
\hline & & BPA3 & 0.1 & & \\
\hline & & BPA30 & 5.8 & & \\
\hline & & BPA100 & 6.5 & & \\
\hline & BPA300 & 12.3 & & \\
\hline$\dot{v}$ & & BPA0.3 & 2.0 & 4.63 & \\
\hline & BPA3 & 0.9 & & \\
\hline & & BPA30 & 6.2 & & \\
\hline & & BPA100 & 9.9 & & \\
\hline & & BPA300 & 32.7 & & \\
\hline & & & & & \\
\hline
\end{tabular}

$\left[\dot{p}_{M}\right],\left[E_{G}\right]$ and $\dot{v}$ are parameters of the DEB described as volume-specific somatic maintenance, specific cost for structure, and energy conductance respectively. $\dot{k}_{r}$ is the recovery rate assumed identical between treatments $\left(\mathrm{d}^{-1}\right) \cdot s_{\text {ini }}$ is the initial intensity of the stress, specific to each level of exposure (-). 
Table 5 Predicted age at birth $\left(a_{b}\right)$, age at metamorphosis $\left(a_{j}\right)$, size at birth $\left(L_{b}\right)$, size at metamorphosis $\left(L_{j}\right)$ and acceleration factor $\left(s_{M}\right)$, when energy conductance $(i)$ is increasing to reach control value following parameters in table $4-8.5^{\circ} \mathrm{C}$-.

\begin{tabular}{|l|c|c|c|c|c|c|c|}
\hline & \multicolumn{3}{|l|}{ Experiment 1 $f=0.7)$} & \multicolumn{2}{l|}{$\begin{array}{l}\text { Experiment } 2(f= \\
0.6)\end{array}$} \\
\hline & BPA0.3 & BPA3 & BPA30 & BPA100 & BPA300 & BPA3 & BPA30 \\
\hline$a_{b}(\mathrm{dpf})$ & 66 & 65 & 68 & 70 & 84 & 65 & 68 \\
\hline$a_{j}(\mathrm{dpf})$ & 141 & 139 & 144 & 147 & 168 & 141 & 145 \\
\hline$L_{b}(\mathrm{~cm})$ & 0.218 & 0.217 & 0.217 & 0.217 & 0.211 & 0.218 & 0.218 \\
\hline$L_{j}(\mathrm{~cm})$ & 0.567 & 0.564 & 0.565 & 0.563 & 0.56 & 0.565 & 0.56 \\
\hline$s_{M}(-)$ & 2.60 & 2.60 & 2.61 & 2.60 & 2.65 & 2.59 & 2.56 \\
\hline & & & & & & & \\
\hline
\end{tabular}



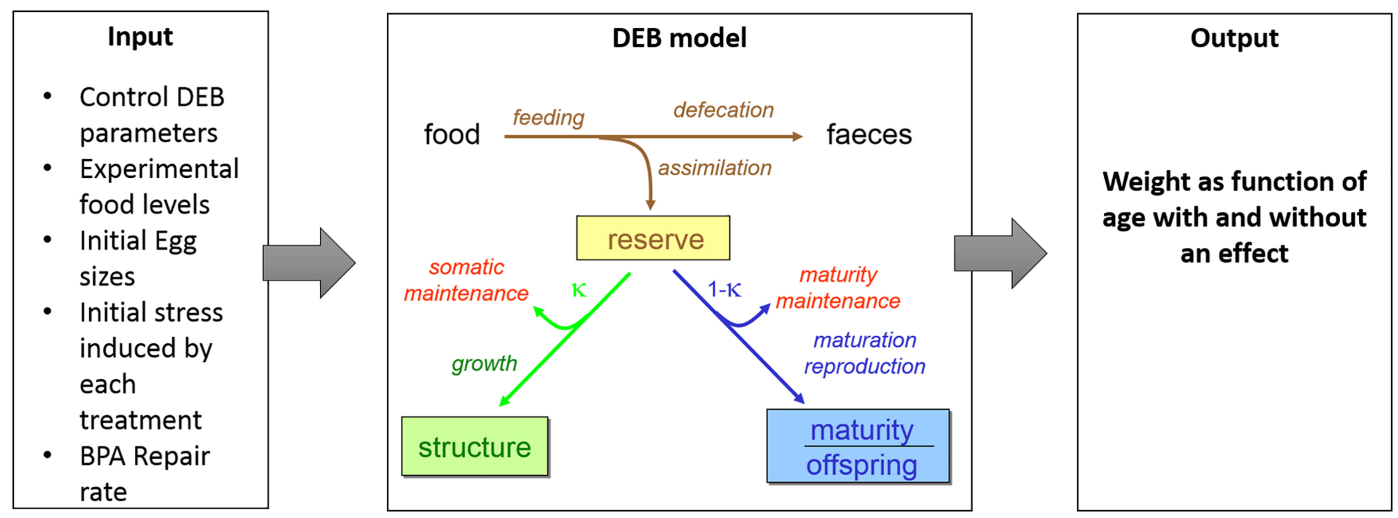

Figure 1 
a

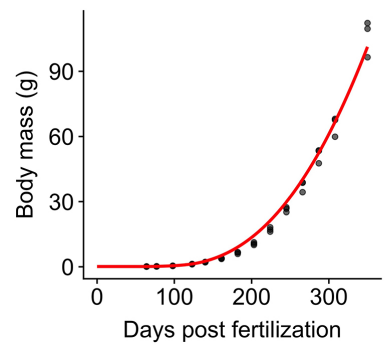

b

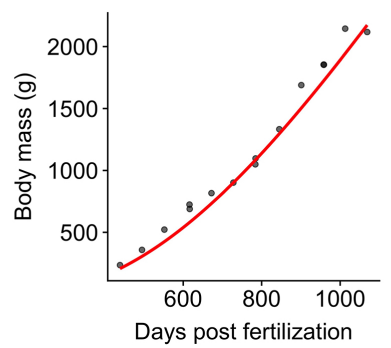

c

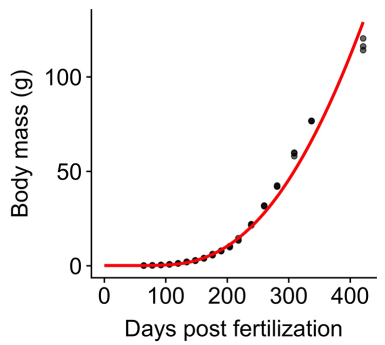

Figure 2 
a

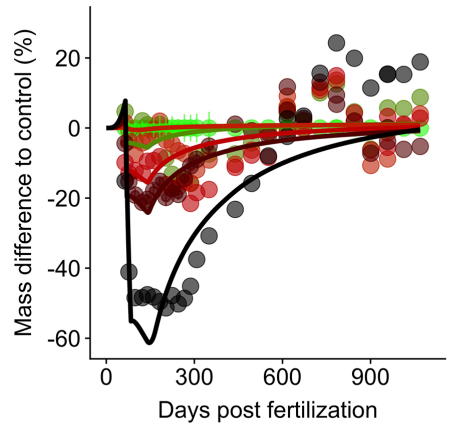

b

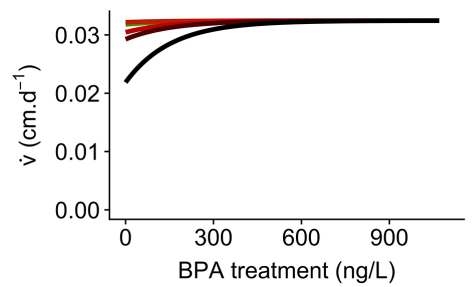

condition

control

- BPA0.3

BPA3

BPA30

BPA100

- BPA300

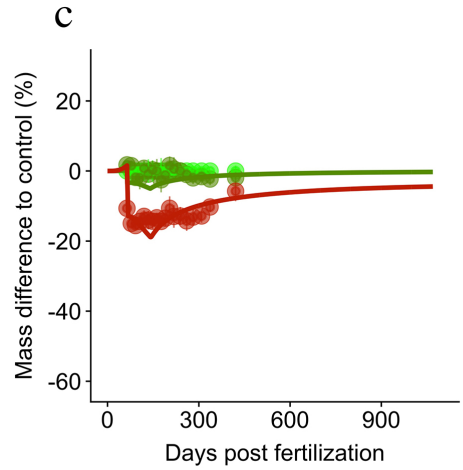

condition

control

- BPA3

- BPA30
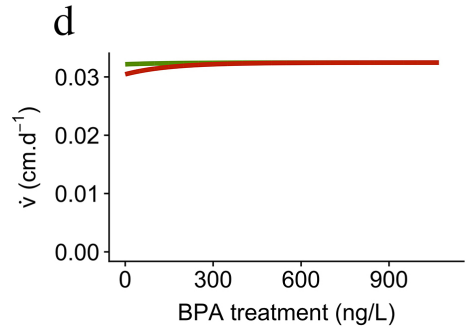

Figure 3 


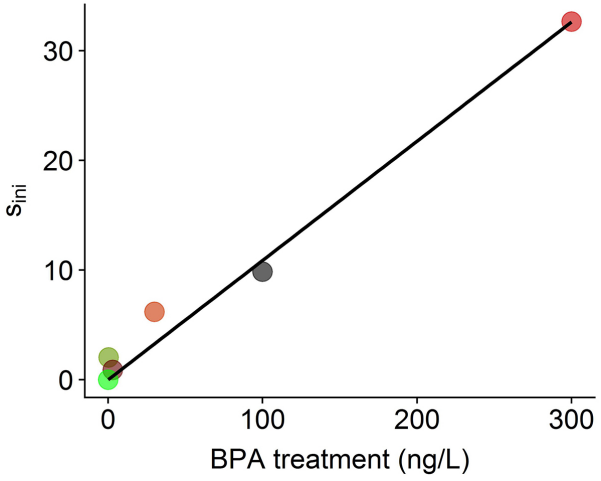

Figure 4 

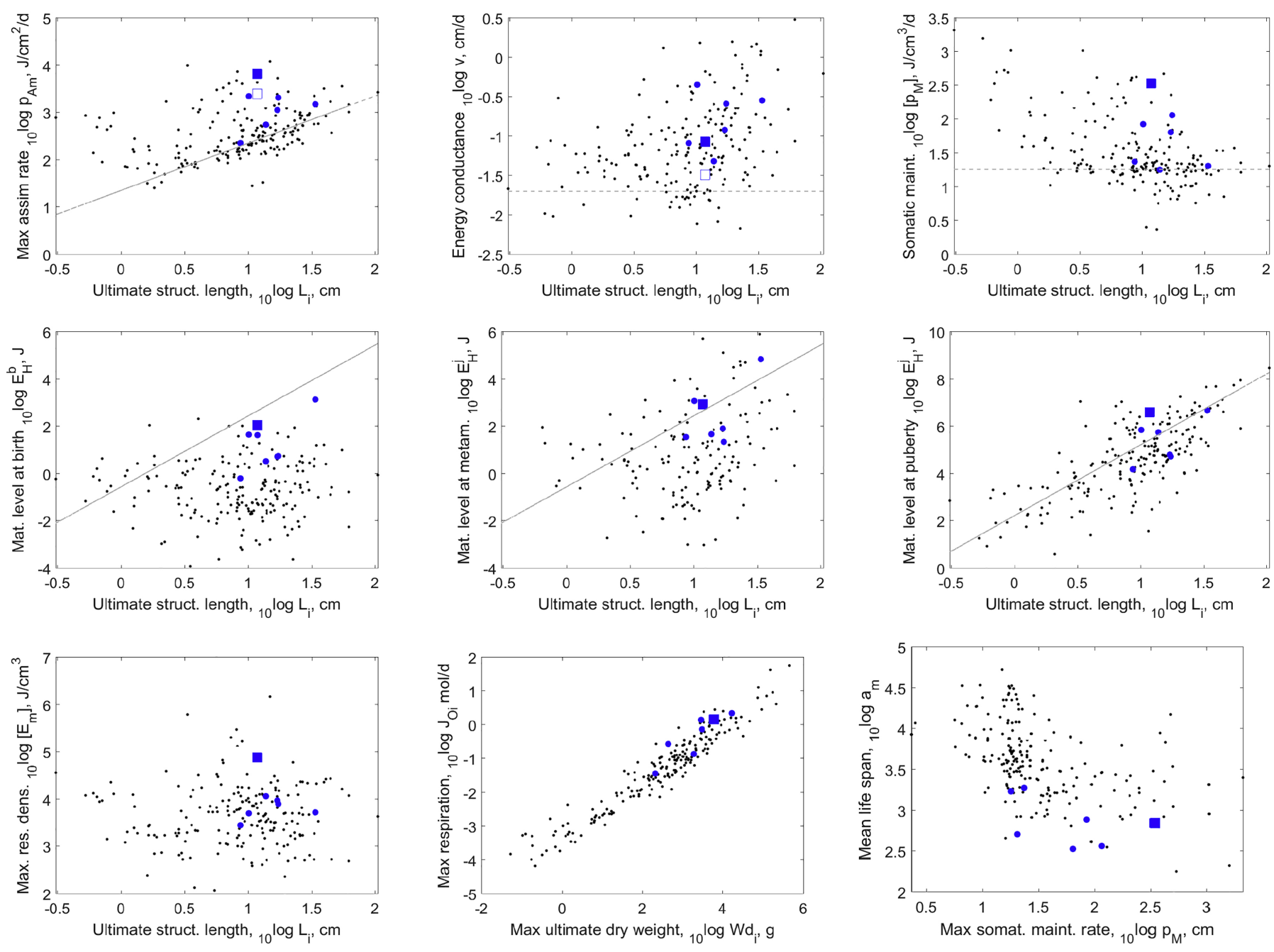

Figure 5 